\title{
Editorial
}

\section{Is it useful to divide level VA of the neck into two sections?}

\author{
Alessandra Rinaldo, M.D., K. Thomas Robbins*, M.D., F.R.C.S.C., F.A.C.S., Alfio Ferlito, M.D., \\ F.R.C.S.(ED.), F.R.C.S., F.A.S.C.P., F.R.C.S. (GLASG.), M.C.A.P., F.R.C.S.I., M.R.C.PATH
}

The lymph nodes of the neck can be divided into six levels. ${ }^{1,2}$ Recently, the American Academy of Otolaryngology-Head and Neck Surgery and the Committee for Head and Neck Surgery and Oncology recommended that levels I, II and V be sub-divided into the respective sublevels of A and B. In other publications ${ }^{3,4}$ we have discussed the importance of the sublevels specific for levels I and II. In this report, we wish to focus on level V, the third region in which the Committee believed there was merit in recognizing sublevels A and B. Level V, also called the posterior triangle, includes the lymph nodes located along the lower half of the spinal accessory nerve and the lymph nodes located along the transverse cervical artery. The supraclavicular nodes, also included in the posterior triangle group, lie in the medial aspect of sublevel VB, some of which may extend below the superior border of the clavicle. An exception to this is the Virchow node, which is located in level IV.

The superior boundary of level $\mathrm{V}$ is in the form of an apex created by the convergence of the sternocleidomastoid and trapezius muscles. The inferior boundary is the clavicle, the anterior (medial) boundary is the posterior border of the sternocleidomastoid muscle and the posterior (lateral) boundary is the anterior border of the trapezius muscle. The dividing line between sublevel $\mathrm{VA}$ and sublevel VB arbitrarily is defined by a horizontal plane corresponding to the inferior border of the anterior cricoid arch. Thus, sublevel VA includes the spinal accessory nodes, whereas sublevel VB includes the nodes following the transverse cervical vessels and the supraclavicular nodes.

The posterior triangle nodes are at greatest risk of harbouring metastases from cancers arising from the nasopharynx, oropharynx (soft palate, oropharyngeal wall), parotid gland, thyroid gland, and cutaneous structures of the posterior scalp and neck. With the exception of thyroid cancer, metastases from these sites are confined to level VA (inferior spinal accessory nodes). On the contrary, metastases from thyroid cancer to level V may occur in sublevel VB (transverse cervical artery nodes) or, less often, to sublevel VA. In general, metastatic nodes of the lower posterior triangle (sublevel VB) carry a higher risk of developing distant metastases. In 2002, Hamoir et al. ${ }^{5}$ advocated the subdivision of level VA into two sections: the apex of level VA or level VAS (superior) and level VAI (inferior), demarcated by the lower two-thirds of the spinal accessory nerve. Having failed to find any apical nodes in a series of neck dissection specimens, the authors concluded that the dissection of level VAS is not necessary in most head and neck cancers. An exception would be for selected patients with skin cancer of the posterior cephalic area (retroauricular region, occipital scalp). This proposal by Hamoir et $a l .{ }^{5}$ for subdividing level VA into two additional zones warrants further consideration by the Committee for Head and Neck Surgery and Oncology of the American Academy of Otolaryngology-Head and Neck Surgery in future deliberations. To avoid confusion, we recommend the current 2002 classification proposed by the Committee. ${ }^{1}$

It should be noted that the six levels described and made standard by the Academy's Committee were adopted from the description of the level system used by surgeons at the Memorial Sloan-Kettering Cancer Center. Ferlito et al. ${ }^{6}$ published an editorial regarding cervical lymph node levels according to the scheme originally devised at the Memorial Sloan-Kettering Cancer Center and pointed out that the levels indicated were seven and not six. The Committee for Head and Neck Surgery and Oncology of the American Academy of Otolaryngology-Head and Neck Surgery and the American Head and Neck Society prefer to use six levels for the classification of neck dissection. Level VII represents the superior mediastinum and is not within the boundaries of conventional neck dissections. With regard to using sublevels to denote specific locations for lymph nodes, we believe there is validity for this and wish to promote an educational process for its recognition. Whereas 
levels I, II and V are divided into A and B sublevels, currently, levels III, IV and VI are not. Future modifications could be considered, such as the separation of level IV nodes into IVA and IVB, as previously proposed by Robbins. ${ }^{7}$ New modifications should be done through consensus of a committee to avoid confusion and facilitate inter-institutional communication. Suggestions for continued improvement and refinements of the present classification should be considered when the classification is revisited by the Academy's Committee in the future. We currently believe it is preferable to describe the levels of the neck using the levels and terminology that have been accepted internationally. The present classification provides a better understanding and communication among head and neck surgeons, radiologists, radiotherapists and pathologists. It also offers an acceptable and important standard for reporting and comparing results from different teams and centres.

\section{References}

1 Robbins KT, Clayman G, Levine PA, Medina J, Sessions R, Shaha A, et al. American Head and Neck Society; American Academy of Otolaryngology-Head and Neck Surgery. Neck dissection classification update: revisions proposed by the American Head and Neck Society and the American Academy of Otolaryngology-Head and Neck Surgery. Arch Otolaryngol Head Neck Surg 2002;128:751-8

2 Lallemant B, Mallet Y, Ala-Eddine C, Lartigau E, Lefebvre JL. La classification radio-chirurgicale des niveaux ganglionnaires cervicaux. Ann Otolaryngol Chir Cervicofac 2003;120:216-24
3 Rinaldo A, Robbins KT, Ferlito A. The importance of distinguishing between sublevel IA and IB in neck dissection. ORL J Otorhinolaryngol Relat Spec 2004;66: 53-5 (Editorial)

4 Talmi YP, Rinaldo A, Robbins KT, Ferlito A. Why promote an educational process for separating level II into level IIA and level IIB in neck dissection? ORL J Otorhinolaryngol Relat Spec (in press) (Editorial)

5 Hamoir M, Desuter G, Grégoire V, Reychler H, Rombaux P, Lengelé B. A proposal for redefining the boundaries of level $\mathrm{V}$ in the neck. Is dissection of the apex of level $\mathrm{V}$ necessary in mucosal squamous cell carcinoma of the head and neck? Arch Otolaryngol Head Neck Surg 2002;128:1381-3

6 Ferlito A, Robbins KT, Medina JE, Shaha AR, Som PM, Rinaldo A. Is it time to eliminate confusion regarding cervical lymph nodes levels according to the scheme originated at the Memorial Sloan-Kettering Cancer Center? Acta Otolaryngol 2002;122:805-7 (Editorial)

7 Robbins KT. Classification of neck dissection: current concepts and future considerations. Otolaryngol Clin North Am 1998;31:639-55

Address for correspondence:

Alfio Ferlito, M.D., F.R.C.S.(Ed.), F.R.C.S., F.A.S.C.P.,

F.R.C.S. (Glasg.), M.C.A.P., F.R.C.S.I., M.R.C.Path,

Director of the Department of Surgical Sciences,

Professor and Chairman of the ENT Clinic,

University of Udine,

Policlinico Universitario,

Piazzale S. Maria della Misericordia,

I-33100 Udine, Italy.

Fax: +390432 559339

E-mail: a.ferlito@uniud.it

Professor A. Ferlito takes responsibility for the integrity of the content of the paper.

Competing interests: None declared 